\title{
Breathing Life Into Dead-Zones
}

\author{
Orkan M. Umurhan ${ }^{1,2, a}$, Richard P. Nelson ${ }^{2}$, and Oliver Gressel ${ }^{3}$ \\ ${ }^{1}$ University of California, Merced \\ ${ }^{2}$ Queen Mary University of London \\ ${ }^{3}$ NORDITA
}

\begin{abstract}
The terrestrial planet formation regions of protoplanetary disks are generally sufficiently cold to be con- sidered non-magnetized and, consequently, dynamically inactive. However, recent investigations of these so-called "Dead-Zones" indicate the possibility that disks with strong mean radial temperature gradients can support instabilities associated with disk-normal gradients of the basic Keplerian shear profile. This process, known as the Goldreich-Schubert-Fricke (GSF) instability, is the instability of short radial wavelength inertial modes and depends wholly on the presence of vertical gradients of the mean Keplerian (zonal) flow. We report here high resolution fully nonlinear axisymmetric numerical studies of this instability and find a number of features including how, in the nonlinear saturated state, unstable discs become globally distorted, with strong vertical oscillations occurring at all radii due to local instability. We find that nonaxisymmetric numerical experiments are accompanied by significant amounts angular momentum transport $(\alpha \sim 0.001)$. This instability should be operating in the Dead-Zones of protoplanetary disks at radii greater than $10-15 \mathrm{AU}$ in minimum mass solar nebula models.
\end{abstract}

\section{Introduction}

Terrestrial planet forming regions of young circumstellar disks (protoplanetary disks, "pp-disks" for short) are thought to harbor vast regions which are magnetically inactive and are, as such, considered to be "dead" [2,7]. This terminal pronouncement is generally accepted to be the case since, to date, no mechanism for non-magnetic instability has been identified and shown to be sustained for stratified fluids under disk-like conditions. The situation in Dead-Zones may be, in fact, quite the opposite with recent reports of finite-amplitude critical layer instabilities showing sustained generation of vortices in Keplerian shear flows (see the work of Marcus, this volume). We too report, in our paper currently in review [11] (NGU2012 hereafter), the results of highly resolved simulations of pp-disks demonstrating how the so-called Goldreich-Schubert-Fricke instability ("GSF") operates by driving strong hydrodynamic activity and produces significant amounts of angular momentum transport.

\section{What is the GSF?}

The GSF instability is a generalized Rayleigh criterion for the instability of axisymmetric flows. Originally proposed as a possible source of instability in the radiative zones of stars [8], it is a viable mech-

\footnotetext{
a e-mail: oumurhan@ucmerced.edu
}

This is an Open Access article distributed under the terms of the Creative Commons Attribution License 2.0, which permits unrestricted use, distribution, and reproduction in any medium, provided the original work is properly cited. 


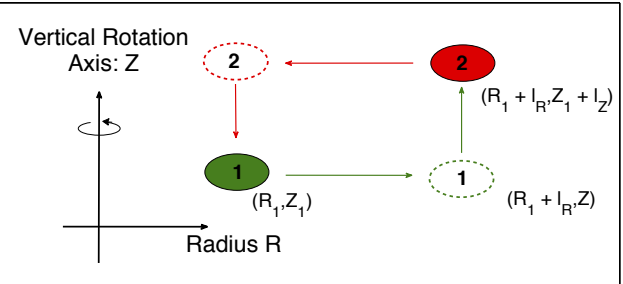

Figure 1. The right panel depicts a cartoon schematic of the instability. Axisymmetric disturbances means that parcels conserve their specific angular momenta. An interchange of parcels 1 and 2 results in a lower total energy if the criterion in Equation 1 results in lower energy and, hence, instability. The quantities $\ell_{R}$ and $\ell_{Z}$ are therefore interpreted as the corresponding wavelengths of disturbances. The instability renders unstable inertial (nearly incompressible) modes.

anism for disks as its only required ingredient is a global radial temperature gradient. In particular, if a model disk in steady state supports a temperature profile of the form $T_{g}(R)=T_{0}\left(R / R_{0}\right)^{q}$ (where $R$ is cylindrical radius), it follows that this sets up a steady state azimuthal flow profile $(\Omega)$ that is no-longer constant on cylinders - typifying the state of affairs for baroclinic conditions. Solving the steady state equation ("thermal-wind" balance) results in $\Omega(R, Z) \approx \Omega_{K}(R)\left[1+0.25 q \epsilon^{2}\left(Z / H_{0}\right)^{2}\left(R / R_{0}\right)^{-2}\right]$, with $Z$ the disk-normal coordinate and where $\Omega_{K}=\Omega_{0}\left(R_{0} / R\right)^{3 / 2}$ is the Keplerian profile, $H_{0}$ is the local scale-height and where $\epsilon$ measure the nominal disk thickness $=H_{0} / R_{0}$ where $R_{0}$ is some fiducial radius.

The instability is axisymmetric, rides on the back of inertial (nearly incompressible) modes, and can be understood from simple energy arguments whose limiting form is the Rayleigh criterion of inviscid Taylor-Couette flows. The lynchpin of the argument is that axisymmetric (inertial-mode) disturbances preserve their parcel specific angular momenta. Consider, therefore, two parcels labelled as " 1 " and " 2 " whose respective coordinate positions in the disk are labeled by $(R, Z)$ and $\left(R+\ell_{R}, Z+\right.$ $\ell_{Z}$ ). Consider the total-kinetic energy of these two parcels in the steady-state configuration and this $E_{0}$. A simple calculation shows that the total energy of the two parcels with their positions switched (Fig. 1) results in a new energy $E_{N}$ whose difference against the original configuration, $\Delta E \equiv E_{n}-E_{0}$ is given by, the corresponding Solberg-Hoiland condition [10]

$$
\Delta E \sim \ell_{R}^{2} \frac{\partial \ln R^{2} \Omega}{\partial \ln R}-\ell_{Z} \ell_{R} \frac{\partial \ln R^{2} \Omega}{\partial \ln Z} .
$$

The derivation of the criterion as stated makes explicit use of the conservation of each individual parcel's angular momentum. For $\Delta E<0$ the configuration, being in a lower energy state, implies such steady states are candidates for linear instability. For cold disks $\epsilon \ll 1$ it means that the length scales of the "switching" must be such that $\ell_{Z} / \ell_{R} \gg 1$. Note that this is a purely baroclinic effect: were $q=0$ there would be no possibility of instability and the energy measure found in (1) would reduce to the classical prediction of stability for simple, purely rotationally supported, accretion disk flows. The quality of the emergent dynamics expected for $\Delta E<0$ is not obvious at this stage and further investigation is required (next section).

\section{Results of numerical simulations}

We are interested in developing a sense for the outcome of this instability in a simple model system. Using the well-tested numerical code for modeling disks (Nirvana and Nirvana-III, [13]) we analyze the response of the equations of motion in a cylindrical disk-like geometry given by,

$$
\begin{aligned}
\partial_{t} \rho+\nabla \rho \mathbf{u} & =0 \\
\partial_{t} \mathbf{u}+\mathbf{u} \cdot \nabla \mathbf{u} & =-\frac{1}{\rho} \nabla P-\nabla \Phi+v \nabla^{2} \mathbf{u}, \\
\partial_{t} T+\mathbf{u} \cdot \nabla T & =\frac{T_{g}-T}{\tau_{\text {relax }}},
\end{aligned}
$$


with $P=\rho T$ with the aforementioned temperature profile $T_{g}$ as the seed state, $\Phi$ represents the gravitational potential of a central point mass star $\Phi=R_{0} \Omega_{0}^{2} /\left(R^{2}+Z^{2}\right)^{1 / 2}$. To best isolate the dynamical processes present during the emergence of the instability we have used the simple Newton's Law of Cooling type of prescription to model the thermal response of the flow characterized by a cooling time $\tau_{\text {relax }}$. For values of $q=-1$ and $\epsilon=0.05$ the radial length scale of the instability is predicted from a linear stability analysis to be $\sim 0.01 R_{0}[11]$ - so it means we must have very high resolution (at least 10-15 points radially) to "see" the instability in its true state, that is to say, disambiguated from numerical noise. We have run our models with approximately 1500 points radially and several hundred vertically covering up to 5 scale heights (and a similar number azimuthally for non-axisymmetric disturbances covering a $\pi / 8$ radian range). We summarize the main results of our investigations:

1. Instability is observed in the form of growing oscillations. This is predicted by the linear theory calculated in detail in our paper NGU2012.

2. Instability indeed emerges on very short radial length scales, $\sim 0.01 R_{0}$ for $q=-1$. Instability is also insensitive to the types of boundary conditions imposed.

3. The instability manifests itself by creating very short radial scale alternating coherent patterns in all dynamically evolving quantities but, especially, in the vertical velocity. Both axisymmetric and non-axisymmetric numerical experiments indicate that the growth/saturation phases are characterized by odd/even vertical velocity structure with respect to the midplane $Z=0$. See left two panels of Fig. 2.

4. The instability operates for cooling times very short compared to the local orbit times of the disk (right panel of Fig. 2).

5. During the growth phase of the linear stability, the dynamics of the growing mode are in radial geostrophic balance as indicated by the relative content in the radial versus meridional kinetic energies (see left panel of Fig. 3). This means to say that the linear dynamics follow, $2 \Omega_{0} v^{\prime} \sim$ $-(1 / \rho) \partial P^{\prime} / \partial R$, where $v^{\prime}$ and $P^{\prime}$ denote fluctuating azimuthal velocities and pressures. This has implications for theoretical/analytical modelling.

6. Furthermore, the asymptotic linear theory analysis of Equations (2-4) in the limit of very short cooling times (found in NGU2012) shows that the radial length scale of the fastest growing mode $\ell_{R}^{(\max )}$ has a dependence $\sim|q| \pi \epsilon^{2} R_{0}$ and have growth rates $\sim \epsilon|q| \Omega_{0}$. These are observed in the numerical experiments reported with the same time scales of growth.

7. Preliminary investigations of the non-axisymmetric development shows that the instability sustains strong amounts of angular momentum transport yielding effective values of turbulent $\alpha \sim 2 \times 10^{-3}$ (right panel of Fig. 3).

\section{Is the GSF reasonably to be expected in protoplanetary disk Dead-Zones?}

The results of these investigations show that this process may be operative for disks whose radiative timescales (on the scale of the observed instabilities, that is) are short compared to the in situ local orbit times. Clearly we have built these models using a very idealized representation of thermal cooling processes and it would behoove one to model the thermal response with a more realistic radiative diffusion prescription. The question we pose here is simple: does one expect the instability to persist 


\section{EPJ Web of Conferences}
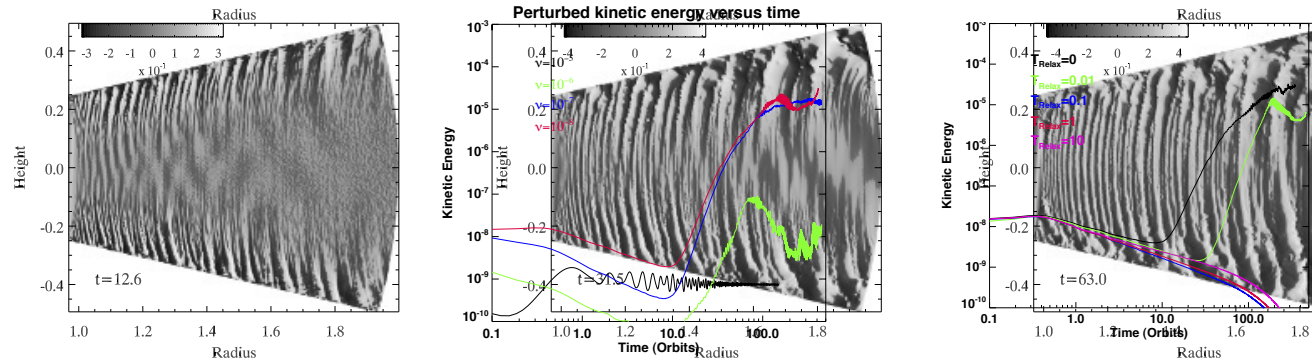

Figure 2: The two left two panels depicts the vertical velocities for axisymmetric simulations at early times $(\mathrm{t}=12.6$, linear growth phase) and later times ( $\mathrm{t}=31.5$, saturation). The former is characterized by breathing modes while the latter states corrugation modes. Times measured in local orbit times at $R=1$. The right panel shows the fate of the response of the disk as a function of the cooling time $\tau$ (shown in the figure as $T_{\text {Relax }}$.)
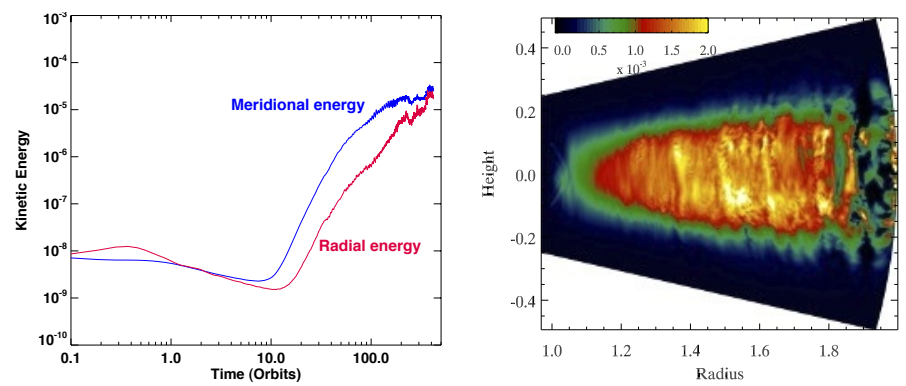

Figure 3. Left panel:relative kinetic energy content of disturbances during growth phase of instability. Right panel: total integrated Reynolds stresses for non-axisymmetric experiments quoted in equivalent values of disk- $\alpha$.

in disk models with radiative diffusion characterised by (integrated) opacities typical of the dust and gas believed to be present in protoplanetary disks? This is certainly the next step in our general course of investigations. However, we can say that for the short radial length scales we see the dominant growing instability, the corresponding thermal/radiative diffusion timescales are indeed much shorter than the local rotation times. The following calculation substantiates this assertion:

According to the standard theory of frequency integrated radiative transfer with Rosseland mean opacities [10] it follows that the thermal timescales due to radiative diffusion is $t_{r}=$ $\Delta^{2}\left(3 C_{p} / 4 a c\right)\left(\rho^{2} \kappa_{R} / T^{3}\right)$, where $\kappa_{R}$ is the Rosseland mean opacities and where $c, a$ and $C_{p}$ are the speed of light, radiation constant and specific heat at constant pressure, and with $\Delta$ measuring the perturbation length scales. Model values of $T$ and $\rho$ representing minimum mass solar models (MMSM) are drawn from the simple irradiated disk models of Chiang \& Goldreich (1997) [1] which compare favorably to realistic disk structure calculations of DAlessio et al. (2001) and Dullemond et al. (2002) $[3,4]$. Using $\rho \approx \rho_{\text {mid }}=2.7 \times 10^{-9}\left(\mathrm{~g} / \mathrm{cm}^{3}\right) F \cdot(R / \mathrm{AU})^{-39 / 14}$ and $T=(R / \mathrm{AU})^{-3 / 7} 120 \mathrm{~K}$ we find that the thermal timescales are (approximately)

$$
t_{r} / P_{\text {orb }}=168 F^{2}\left(\kappa_{R} / \mathrm{cm}^{2} / \mathrm{g}\right)(\Delta / R)^{2}(R / 20 \mathrm{AU})^{-53 / 14}, \quad P_{\text {orb }} \equiv 2 \pi / \Omega_{K}
$$

where $F$ represents the relative mass fraction of the pp-disk to a solar disk. Equating to $\Delta$ the lengthscale of the fastest growing mode, $\ell_{R}^{(\max )}$, and using $q=-3 / 7, \epsilon=0.05$ we find $\Delta / R \approx 0.0034$. From the calculations of Henning \& Stognienko (1996) [9], the opacities in the temperature range 
$50-150 K$ hover below $1-5 \mathrm{~cm}^{2} / \mathrm{gm}$. Using the lower values as fiducial ${ }^{1}$ we find that at $R=10 \mathrm{AU}$ $\leftrightarrow t_{r} / P_{\text {orb }}=0.027 F^{2}$ while at $R=20 \mathrm{AU} \leftrightarrow t_{r} / P_{\text {orb }}=0.002 F^{2}$. We conclude that for the perturbation length scales on which the GSFI is active, the thermal timescales of MMSM pp-disks are sufficiently short to accommodate the process, provided one is investigating disk dynamics out beyond at least $10 \mathrm{AU}$ - a region which is squarely within Dead Zones and the realms in which terrestrial planet formation takes place.

\section{References}

[1] Chiang, E. I., \& Goldreich, P. Astrophys. J.490, 368-376 (1997)

[2] Chiang, E., \& Youdin, A. N. Annual Review of Earth and Planetary Sciences 38 493-522 (2010)

[3] D’Alessio, P., Canto, J., Calvet, N., \& Lizano, S. Astrophys. J. 500411 (1998)

[4] Dullemond, C. P., van Zadelhoff, G. J., \& Natta, A. Astron. Astrophys.389 464 (2002)

[5] Dullemond, C. P., \& Dominik, C. Astron. Astrophys. 434971 (2005)

[6] Fricke, K. Zeit. für Astrophys. 68, 317-332 (1968)

[7] Gammie, C. F. Astrophys. J. 457 355-362 (1996)

[8] Goldreich, P., \& Schubert, G. Astrophys. J. 150 571-588 (1967)

[9] Henning, T., \& Stognienko, R. Astron. Astrophys.311, 291 (1996)

[10] Kippenhahn, R., \& Weigert, A., Stellar Structure and Evolution (Springer-Verlag, Berlin, 1990) 468 pgs.

[11] Nelson, P.P., \& Gressel, O. \& Umurhan, O. M. Mon. Not. Roy. Astron. Soc. in review (arXiv e-print: 1209.2753) (2012)

[12] Pollack, J. B., McKay, C. P., \& Christofferson, B. M. Icarus 64471 (1985)

[13] Ziegler, U. Journal of Computational Physics 2301035 (2011)

\footnotetext{
${ }^{1}$ The frequency averaged opacities calculated in Henning \& Stognienko (1996) [9] assumes a grain mixture of amorphous silicates as typical of the interstellar medium. As part of the general paradigm of long term disk evolution, the sizes of these grains grow quickly $\left(\sim 10^{4} \mathrm{yrs}\right)$ and achieve sizes of about $10^{2}-10^{3} \mu \mathrm{m}$ [5]. With grain size growth comes a reduction of opacities [12]. D'Alessio et al. (1999) argue that spectral energy distributions of T-Tauri stars support the presence of grains $\sim 3 \mathrm{~mm}$. Thus, referencing Figs. 6-7 of Pollack et al. (1985) [12] shows that grains with $300 \mu$ m radii correspond Rosseland Means opacities of $0.2 \mathrm{~cm}^{2} / \mathrm{gm}$ in the temperature range of interest, it is therefore fair to assume a fiducial upper bound for $\kappa_{R}$ of $1 \mathrm{~cm}^{2} / \mathrm{gm}$.
} 